\title{
50 ANS DE TIMBRES SOVIÉTIQUES (1918-1969)
}

\author{
PIERRE HUBERT
}

(Ecole des mines de Paris)

\section{DOMINIQUE LABBÉ}

Revue française de science politique XXIII (6), décembre 1973, pp. 1157-1170.

L'existence, dans une collectivité telle que l'URSS, d'une idéologie fortement structurée et totalisatrice étant admise, son étude se heurte à la multiplicité même de ses manifestations. Le timbre-poste constitue une de ces manifestations, régulièrement émise, où le message idéologique doit être condensé. Dans l'ensemble des quelques 4000 timbres émis en Union soviétique une double coupe, synchronique d'abord en effectuant un classement par thèmes, puis diachronique en découpant le continuum historique en périodes, a permis d'analyser le contenu idéologique des timbres soviétiques ainsi que son évolution de 1918 à 1969. La philatélie pourrait donc peut-être fournir un champ d'investigation au chercheur politique.

\section{YEARS OF SOVIET STAMPS (19184969)}

Given the existence in a community such as the USSR of a highly structured and totalizing ideology, the very multiplicity of ways in -which this is manifested is an obstacle to its study. The postage stamp is one such, regularly issued, manifestation in which the ideological message has to be condensed. Taking all the 4000 stamps issued in the Soviet Union, it was possible to analyse the ideological content and trend of Soviet stamps from 1918 to 1969 by means of a twofold selection based first on a classification by subject matter, and secondly on a division of the historical sequence into periods. Philately might perhaps therefore constitute an area worth investigation by political researchers.

Manuscrit des auteurs.

Toute citation doit se faire à partir de la Revue. 
Nous allons essayer dans cette note de présenter le résumé d'un travail esquissé il y a deux ans et qui donne aujourd'hui quelques résultats intéressants. L'idée de départ : fournir un tableau si possible condensé et synthétique de l'idéologie soviétique depuis 1917 à partir des timbres émis par les postes d'URSS depuis cette date. Cette prétention ressemble à un canular de chercheurs égarés par la passion philatélique. Certes, nous ne chercherons pas à cacher que nous avons été attirés par le côté distrayant, divers et toujours surprenant de ce gigantesque kaléidoscope, mais que l'on nous permette quelques réflexions qui justifieront peut-être notre propos. Ces quelques remarques ont trait d'une part aux problèmes que pose l'étude de l'idéologie dans une collectivité nationale moderne et d'autre part au choix d'un corpus de signes qui permette une analyse quelque peu scientifique.

\section{L'étude de l'idéologie et ses problèmes}

Le concept d'idéologie est l'objet de nombreuses discussions théoriques dans lesquelles il n'est pas possible d'intervenir ici. Dans la perspective purement expérimentale qui est la nôtre nous avons adopté une définition relativement restrictive de l'idéologie :

«Système d'idées et de jugements, explicite et généralement organisé, qui sert à décrire, expliquer, interpréter ou justifier la situation d'un groupe ou d'une collectivité, et qui, s'inspirant largement de valeurs, propose une orientation précise à l'action historique de ce groupe ou de cette collectivité. ${ }^{1}{ }^{»}$

$\mathrm{Au}$ niveau d'une collectivité nationale moderne comme l'URSS, il est aujourd'hui courant d'admettre l'existence d'une idéologie globale fortement structurée et totalisatrice, c'est-à-dire tendant à investir, avec plus ou moine de succès l'ensemble de la vie sociale. Toutefois cette action peut revêtir deux grandes formes. D'une part, il existe un certain nombre de secteurs où l'idéologie est facilement analysable, mesurable, parce qu'elle agit en quelque sorte à découvert. Dans le Parti et l'Etat, dans la presse, la radio-télévision, les discours des dirigeants, etc., les idées, les valeurs, les normes sont explicites. Il n'en est pas de même dans la plus grande part de la vie sociale où l'idéologie passe par de nombreux relais et médiations. Le premier niveau privilégie naturellement l'aspect politique du problème mais en contrepartie il offre l'avantage de se prêter à des analyses plus raffinées, comme, par exemple, l'analyse thématique que nous nous proposons de mener. Ce type de travaux suppose une série de messages explicites dont une étude systématique et exhaustive doit permettre de dégager les thèmes qui en constituent l'ossature. Cependant le chercheur se heurte à un obstacle. Il se trouve affronté à un nombre colossal de signes imprimés ou diffusés dans des périodes très courtes. La plupart du temps, faute de pouvoir extraire des échantillons réellement représentatifs de ces corpus gigantesques il devra «pêcher » à gauche et à droite les messages, citations, etc. qui lui semblent les plus intéressants pour son étude. De ce fait, il risque la

\footnotetext{
${ }^{1}$ ROCHER (G.), Introduction à la sociologie générale, Editions HMH, Paris, 1970, tome III, p. 87.
} 
censure a posteriori qui lui reprochera d'avoir substitué à l'objet réel de l'étude le contenu que sa subjectivité a privilégié de façon consciente ou non. C'est le travers bien connu de la kremlinologie, par exemple.

Ce problème n'est cependant pas toujours insoluble si l'on veut bien considérer qu'il existe un certain nombre de cas où le message doit être concentré au maximum et se trouve émis à intervalles relativement espacés mais réguliers. Par exemple les affiches de propagande, les slogans de manifestations, les mots d'ordre des congrès, les campagnes politiques diverses... La principale caractéristique de ce type de «média » particuliers, quoique souvent marginaux, vient de l'absence d'ambiguïté, d'emphase ou de rhétorique. Le message doit apparaître à l'état pur et surtout le décodage (l'effet souhaité sur le destinataire) doit être immédiat, pour ainsi dire automatique, inconscient.

C'est précisément cette transparence assez bonne qui permet de se livrer à une étude de contenu aisée et relativement productive ${ }^{2}$. Un bon exemple de ce type de recherche sur l'URSS nous est fourni par l'étude de Yakobson et Lasswell sur l'évolution des slogans du premier mai de 1917 à 1943 où ils décelèrent le passage de thèmes internationalistes et révolutionnaires à des idéaux de type patriotiques et proprement soviétiques ${ }^{3}$.

C'est en ce sens que l'on peut prétendre que ces signes offrent, en quelque sorte, un reflet fidèle, un condensé de la ligne politique et de l'idéologie qui la sous-tend.

\section{Avantages spécifiques du timbre poste}

Les considérations d'ordre général qui précèdent nous semblent tout à fait applicables au timbre-poste, avec même un certain nombre d'avantages particuliers. Et tout d'abord celui d'une production relativement homogène par ses caractéristiques physiques et régulièrement étalée dans le temps contrairement aux slogans, discours, affiches, etc. dont la naissance dépend d'événements particuliers et en subissent l'empreinte. Premier intérêt, donc, une représentativité assez bonne de l'espace-temps 1917-1969.

\footnotetext{
${ }^{2}$ L'analyse de contenu peut se définir comme une « technique de recherche pour la description objective, systématique et quantitative du contenu manifeste des communications » (BERELSON (B.), Content Analysis in Communication Research, Glencoe, The Free Press, 1952, p. 18). Il est important de souligner que la recherche doit porter sur le contenu manifeste. Le principal avantage de cette forme d'approche est de rompre avec la dichotomie idéologie affirmée/idéologie latente. L'analyse de contenu postule que le message est un, au-delà des formes particulières. Peu importe que «l'idéologie affirmée » soit par exemple, l'égalité raciale, une analyse des magazines américains révèle un profond racisme qui en est le contenu manifeste, la seule idéologie qu'ils véhiculent. L'autre, celle qui sert de repère, de justification, n'est même pas une fiction, tout juste une absence. (BERELSON, SALTER, "Majority and Minority Americans: An Analysis of Magazine Fiction », Public Opinion Quarterly, 1946, 10, pp. 168-190). L'autre intérêt majeur de la méthode réside dans son aspect systématique. Le corpus est traité en son entier à l'aide de techniques facilement reproductibles, les résultats sinon leur interprétation, doivent être contrôlables et dans la mesure du possible, non discutables. Sur l'analyse de contenu voir principalement : PINTO (R.) et GRAWITZ (M.), Méthodes des sciences sociales, Dalloz, Paris, 1964, pp. 497-539 ; KIENTZ (A.) : Pour analyser les média, l'analyse de contenu, Marne, 1970 ; LASSWELL (H.D.) : «L'analyse de contenu et le langage de la politique », Revue française de science politique, juin 1952, pp. 505-520.
}

${ }^{3}$ YAKOBSON (S.), LASSWELL (H.D.), « Trend : May Day slogans in Soviet Russia » in LASSWELL (H.O.) et LEITES (N.) : Langage of politics, New York, Stewart, 1949. 
Deuxième facilité offerte par ce corpus : son absence d'ambiguïté qui permet un codage relativement exact ${ }^{4}$.

Quant à la distance à établir entre le timbre-poste et l'univers politique, nous ferons remarquer que le simple fonctionnement des services postaux n'exigerait qu'un nombre restreint de valeurs postales susceptibles, à la manière d'une boîte de poids, de réaliser les divers affranchissements. Or il n'en est jamais ainsi. Les postes de tous les pays, postes dont les pouvoirs politiques ne se désintéressent jamais, éditent des séries toujours plus nombreuses de timbres variés. L'URSS n'échappe pas à cette inflation. Bien sûr, cette pratique peut se justifier de diverses manières. Souci d'enjoliver le travail des fonctionnaires ou de pédagogie envers la clientèle ou encore moyen commode d'éponger une partie des liquidités monétaires excessives mises imprudemment en circulation dans les Etats modernes de l'Ouest comme de l'Est. Il n'en reste pas moins que ces vignettes passent par de nombreuses mains en particulier enfantines et forment une sorte d'imagerie officielle dont la souplesse est évidente et les effets non négligeables. Que l'on songe par exemple à l'imagerie africaine de l'époque coloniale largement répandue souvent grâce aux timbres et aux images ornant les tablettes de chocolats... De nombreux indices peuvent renforcer cette thèse. Ainsi, la manie de la «commémoration » qui n'a épargné aucun de nos grands hommes. D'ailleurs nous verrons que les timbres d'URSS ont eux aussi des thèmes aux résonnances profondément politiques.

Nous nous sommes donc penchés sur les 3841 timbres émis en URSS par les postes fédérales de 1917 à $1969^{5}$ et nous avons effectué dans cet ensemble une double coupe. Synchronique d'abord, suivant la méthode du classement par thèmes, puis diachronique, en découpant le continuum historique en une série de périodes.

Il ne faudrait pas que le lecteur s'attende à trouver des révélations sur l'URSS. Grosso-modo, nous nous sommes contentés de retrouver les traits et les variations les plus marquants de l'idéologie soviétique. Ces résultats sont résumés ici non pas simplement pour eux-mêmes mais plutôt pour illustrer les possibilités de la méthode mise au point.

\section{MÉTHODE}

Dans un premier temps nous avons donc cherché à classer nos timbres suivant le ou leurs thèmes principaux. A chaque timbre nous avons affecté un ou plusieurs mots clefs caractérisant le ou les thèmes, quelquefois sous-thèmes, évoqués par eux ${ }^{6}$. Nous avons retenu quinze thèmes principaux à partir de la classification thématique effectuée par les rédacteurs

\footnotetext{
${ }^{4}$ Voir la discussion de ce problème dans la partie consacrée à la méthode utilisée.

${ }^{5}$ Catalogue des timbres postaux de l'URSS, 1918-1969, Moscou, Agence centrale philatélique, « Sayouz Pietchat $», 1970$.

${ }^{6}$ Dans l'analyse thématique d'origine (Propp, Tomachevski, etc.) le thème est le noyau de la phrase (sujet + prédicat). Repris par l'analyse de contenu anglo-saxonne, le principe a toutefois éclaté en diverses branches et la notion de thème s'est amplifiée. Cependant, le principe de l'analyse se retrouve, relativement inchangé. De même que le «thème » des formalistes russes est l'unité de base de la littérature, on peut repérer des " unités d'information », base du discours journalistique, (Violette MORIN : L'écriture de presse, Paris, Mouton, 1969, pp. 25-31) des mots clefs dont le repérage met à jour les tendances, les préoccupations, de ceux qui les utilisent. (Par exemple, Louis QUESNEL : «Les mots à majuscule en politique », Communications et langages, 3-1969.)
} 
du catalogue philatélique d'Union soviétique. Nous les énumérons ici en leur associant le nombre de timbres qui leur ont été affecté (tableau I).

TABLEAU 1 : Principaux thèmes avec le nombre de timbres qui leur sont affectés

\begin{tabular}{|l|l|l|l|}
\hline Thèmes & Effectifs & Thèmes & Effectifs \\
\hline Moscou-URSS & 890 & Révolution d'Octobre & 298 \\
\hline Littérature-Arts & 845 & PC de l'URSS & 250 \\
\hline Economie nationale & 596 & Deuxième guerre mondiale & 230 \\
\hline Sciences-Techniques & 491 & Jeunesse & 197 \\
\hline Relations internationales & 482 & Nature & 188 \\
\hline Marx-Engels-Lénine & 427 & Histoire russe & 176 \\
\hline Armée-Décorations & 305 & Femmes & 149 \\
\hline Sports & 303 & Moyenne & 388 \\
\hline
\end{tabular}

Cette classification appelle quelques remarques :

1. Comme document de base nous avons utilisé l'index situé à la fin du catalogue. Un examen minutieux nous a montré que cette classification n'avait pratiquement pas à être rectifiée tant au niveau de l'affectation des timbres qu'à celui des mots clefs. Ceci n'a rien d'étonnant si l'on se souvient de la remarque que nous faisions plus haut concernant le manque d'ambiguïté nécessaire du message au moins dans son thème principal. Afin d'alléger l'analyse, quelques regroupements ont été effectués, (par exemple «Armée rouge » et « décorations) ou « MarxEngels » et «Lénine », ce dernier étant d'ailleurs nettement prépondérant). Nous avons conservé les anciennes catégories au titre de sous-thèmes afin de pouvoir réintroduire des distinctions au moment pertinent. Les quelques ambiguïtés non flagrantes ont été conservées en vertu du principe que l'idéologie peut aussi se dévoiler par son travail propre. (Par exemple, nous n'avons pas construit de catégorie Staline non seulement à cause du peu de timbres qui lui sont consacrés mais aussi parce qu'ils se trouvent tous classés dans « PCUS $\gg \ldots$ !).

2. On aura remarqué que certains timbres sont classés plusieurs fois puisque le total du classement par thèmes est supérieur au nombre de timbres émis depuis 1917. Beaucoup d'entre eux n'ont qu'un seul thème, mais presque la moitié en ont deux, quelques-uns trois, même quatre. Par exemple, le premier timbre émis après juin 1941 - « Tu seras un héros » - se trouve à la fois dans «jeunesse » et dans « seconde guerre mondiale » (illustr. 1). 


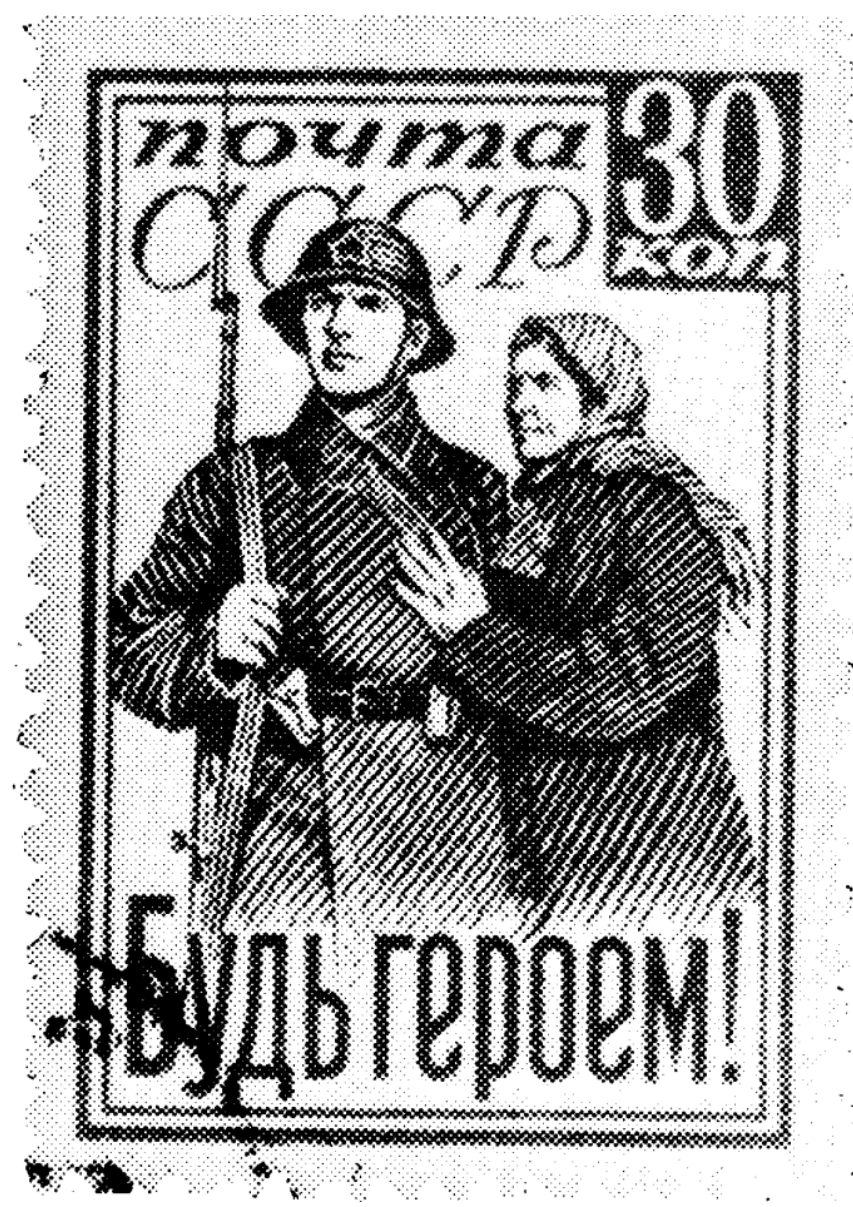

Illustration 1. «Tu seras un héros ! » II s'agit du premier timbre émis après l'entrée en guerre de l'Union soviétique en 1941.

Mais, les thèmes ne sont pas uniformément répartis sur l'ensemble de la période 1917-1969. $\mathrm{Au}$ contraire leurs parts relatives sont en constante évolution et celle-ci ne manque pas de signification. Afin de la saisir dans ses traits les plus caractéristiques, nous avons soumis l'ensemble du catalogue à une analyse diachronique.

Le contenu des thèmes étant délimité avec un minimum de précision, nous allons dans un deuxième temps chercher à fixer l'évolution historique de l'ensemble. Pour cela, nous avons divisé le continuum historique 1917-1969 en six périodes, à partir de six des événements majeurs qui ont marqué l'histoire soviétique (Tableau 2).

TABLEAU 2 : Les six périodes et leurs délimitations

Période I. De la Révolution d'Octobre à la mort de Lénine (21 janvier 1924) 194 timbres,

2. De la mort de Lénine à la deuxième guerre mondiale (22 juin 1941) 613 timbres.

- 3. La deuxième guerre mondiale (8 mai 1945)

165 timbres.

- 4. De la fin de la guerre au XXe Congrès du PCUS (février 1956)

892 timbres.

- 5. Du XXe Congrès à la chute de Khrouchtchev (14 octobre 1964)

1253 timbres.

6. De la chute de Khrouchtchev à la fin de 1969

724 timbres. 
Il y a certes beaucoup à dire sur les événements retenus et, comme tout découpage, celui-ci comporte une part d'arbitraire. Chacune de ces périodes comporte au moins un avantage certain : une population de timbres suffisante pour être traitée statistiquement, leur inégalité n'étant pas un obstacle dans une perspective comparative. Un certain nombre d'outils ont été élaborés afin de mesurer les modifications internes du corpus avec un maximum de clarté et de synthèse.

Fréquence : nous définirons la fréquence d'un thème pour une période comme le rapport entre le nombre de timbres auxquels ce thème a été affecté et le total de la période considérée.

Spectre : le spectre d'un thème sera l'histogramme des fréquences de celui-ci pour les différentes époques (fig. 1 à 15).

Fréquence totale : la fréquence totale d'un thème sera le rapport du nombre total de timbres émis auxquels ce thème aura été affecté au total de timbres émis.

Tableau de synthèse : enfin pour donner une représentation schématique de l'ensemble nous affecterons chaque période de chaque thème d'un indicateur égal à 1 si la fréquence de ce thème pour la période considérée est supérieure à la fréquence totale, égal à 0 dans le cas contraire ${ }^{7}$. L'ensemble de ces indicateurs a été rassemblé dans le tableau 3 qui figure en quelque sorte la structure d'ensemble du corpus.

Tableau 3. Tableau de synthèse : indicateur de sur-emploi (1) ou sous-emploi (0) du thème dans chaque période.

\begin{tabular}{|c|c|c|c|c|c|c|c|c|c|c|c|c|c|c|c|}
\hline PERIOOES &  & & 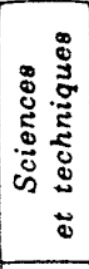 & 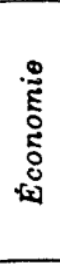 & 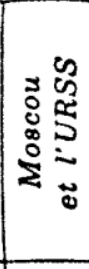 & 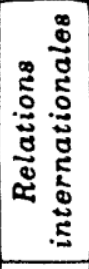 & 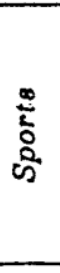 & 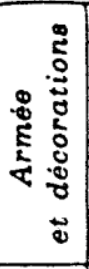 & 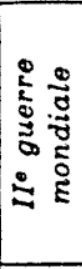 & 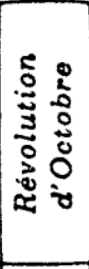 & 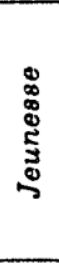 & $\begin{array}{l}0 \\
\vdots \\
\vdots \\
z \\
z\end{array}$ & $\begin{array}{l}2 \\
\text { S } \\
\text { s }\end{array}$ & 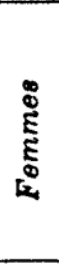 & 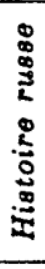 \\
\hline $\begin{array}{c}1 \\
1917-1924 \\
\end{array}$ & 1 & 0 & 0 & 0 & 0 & 0 & 0 & 0 & 2. & 1 & 0 & 0 & 0 & 0 & 0 \\
\hline \begin{tabular}{|c|}
2 \\
$1924-1941$
\end{tabular} & 0 & 1 & 0 & 1 & 1 & 0 & 0 & 0 & 2. & 1 & 0 & 0 & 1 & 0 & 1 \\
\hline \begin{tabular}{|c|}
3 \\
$1941-1945$
\end{tabular} & 1 & 1 & 0 & 0 & 0 & 0 & 0 & 1 & 1 & 0 & 1 & 0 & 0 & 0 & 1 \\
\hline \begin{tabular}{|c|}
4 \\
$1945-1956$
\end{tabular} & 0 & 1 & 0 & 1 & 1 & 0 & 0 & 1 & 0 & 0 & 0 & 0 & 1 & 0 & 1 \\
\hline \begin{tabular}{|c|}
5 \\
$1956-1964$
\end{tabular} & 1 & 0 & 1 & 0 & 1 & 1 & 1 & 0 & 0 & 0 & 1 & 1 & 0 & 1 & 0 \\
\hline $\begin{array}{c}\mathbf{6} \\
1964-1969\end{array}$ & 0 & 0 & 1 & 0 & 1 & 1 & 1 & 1 & 1 & 0 & 0 & 1 & 0 & 0 & 0 \\
\hline
\end{tabular}

\section{LA THÉMATIQUE POSTALE}

En ce qui concerne l'importance numérique de chacun des thèmes telle qu'elle apparait dans le tableau 1 , on ne trouvera rien de très étonnant, si ce n'est la part des relations internationales,

\footnotetext{
7 Le principe même de cet indicateur est sa brutalité. La dichotomie n'est pas entre présence et absence mais entre présence relativement forte (1) ou relativement faible (0). Une lecture rapide de chaque ligne du tableau permet de repérer les préoccupations dominantes de chaque époque et, en creux, le déclin d'autres thématiques.
} 
l'URSS ayant la réputation d'être refermée sur elle-même, et d'autre part la faiblesse relative des thèmes «Révolution d'Octobre » et surtout «Parti communiste » qu'on croirait volontiers omniprésents. Ce classement général ne fait que heurter ou conforter des idées reçues. Plus intéressante nous a semblé l'étude interne des différents thèmes et l'évolution de leur part relative dans l'ensemble de la production postale (analyse diachronique),

La plupart de ces grandes catégories est riche de nuances, de sous-thèmes qui lui donnent de nouvelles dimensions par la multitude des connotations qui s'y attachent et que nous allons très rapidement passer en revue dans ses aspects les plus caractéristiques en regroupant l'ensemble en trois grands groupes de problèmes.

\section{L'héritage du marxisme et du bolchevisme}

Si l'on considère la façon dont apparaît cet héritage dans les timbres postes on s'aperçoit qu'il tombe quelque peu en oubli. Seuls « Marx-Engels-Lénine » connaissent une fréquence un peu supérieure à la moyenne. Encore faut-il remarquer que Lénine fournit le plus fort du contingent. Le premier timbre de cette série date de la mort de Lénine. Il s'agit d'une image très simple, son portrait le plus répandu (illustr. 2). Par la suite, il figurera sous des formes différentes ; principalement de plain-pied, personnage lointain, d'aspect figé, qui drapé dans sa redingote fixe l'horizon (illustr. 3). Ce sont des copies des deux ou trois statues qui ornent de nombreux squares et places du pays.

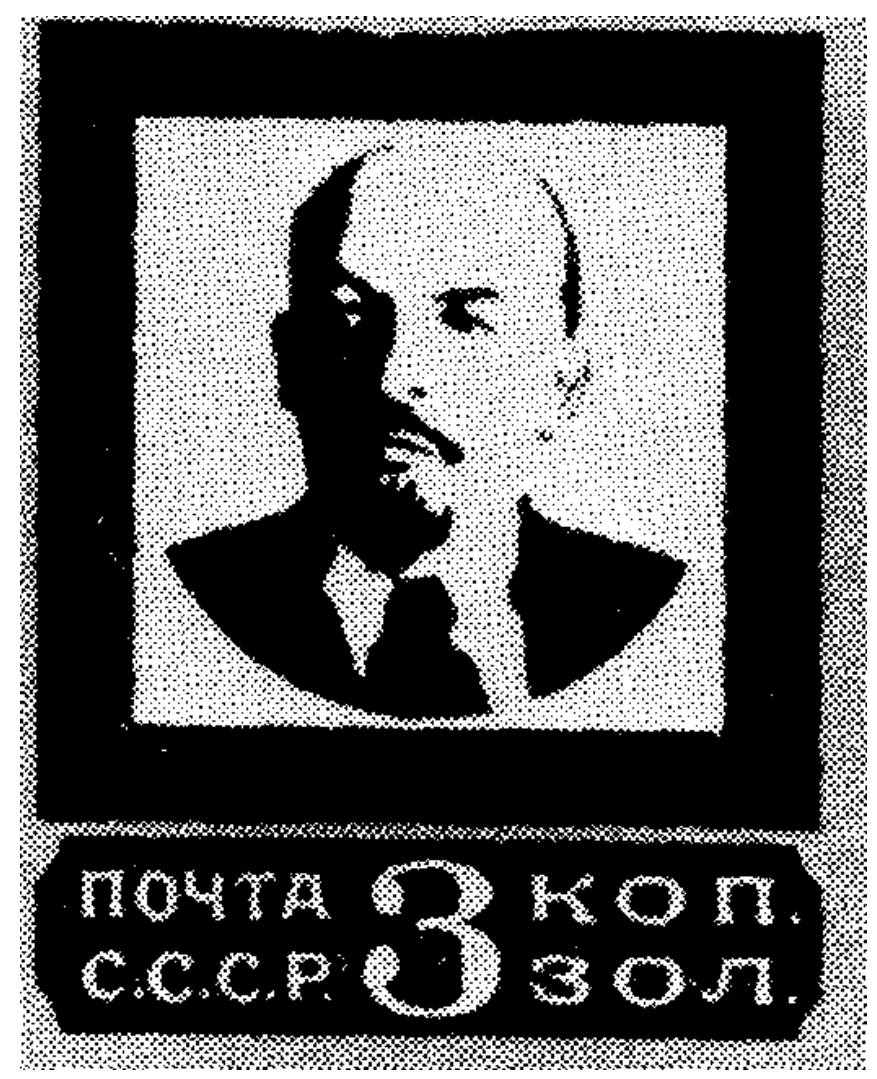

Illustration 2. Le premier timbre consacré à Lénine, en signe de deuil juste après sa mort. 


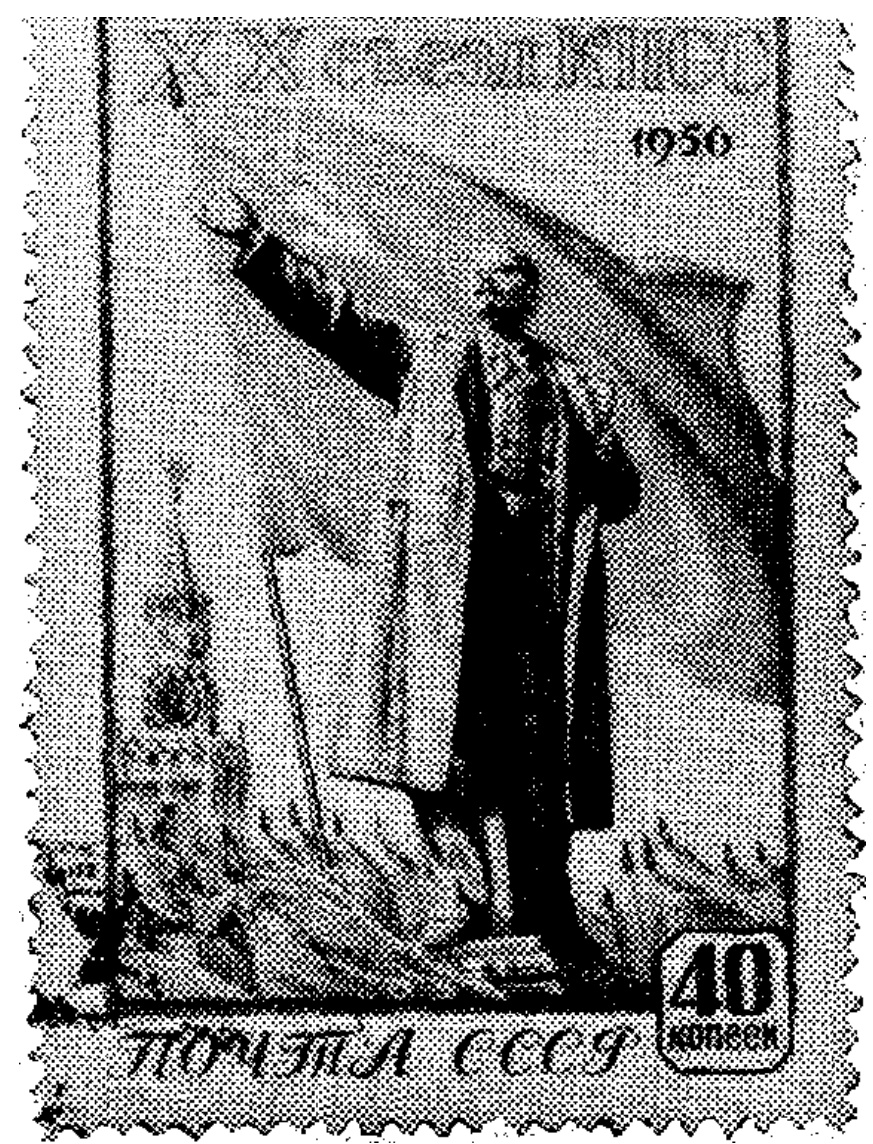

Illustration 3. Timbre commémoratif du $\mathrm{XX}^{\mathrm{e}}$ Congrès du Parti communiste soviétique. Il représente Lénine devant une tour du Kremlin.

II faut ajouter à cela que la plupart de ces portraits sont publiés à l'occasion de dates officielles : fondation du PCUS, congrès, ou bien en commémoration de la Révolution d'Octobre, on s'aperçoit alors que le nombre des vignettes de ce groupe se réduit encore, puisqu'elles entrecroisent souvent deux thèmes. L'absence quasi-complète de référence à la théorie marxiste et aux œuvres (en tout et pour tout trois timbres sont consacrés au centenaire du Manifeste et un pour celui du Capital), fait penser que seul Lénine représente, avec la Révolution d'Octobre, l'élément de légitimité majeur du pouvoir du Parti communiste et de sa ligne politique. Cette hypothèse se trouve renforcée par la présence de très nombreuses associations entre Lénine, la Révolution d'Octobre et le PCUS (par exemple le timbre du XXe Congrès). On pourra se convaincre de ces rapports associatifs en remarquant la similarité des profils de leurs spectres respectifs, sauf pendant la guerre (fig. 1 et 2). 


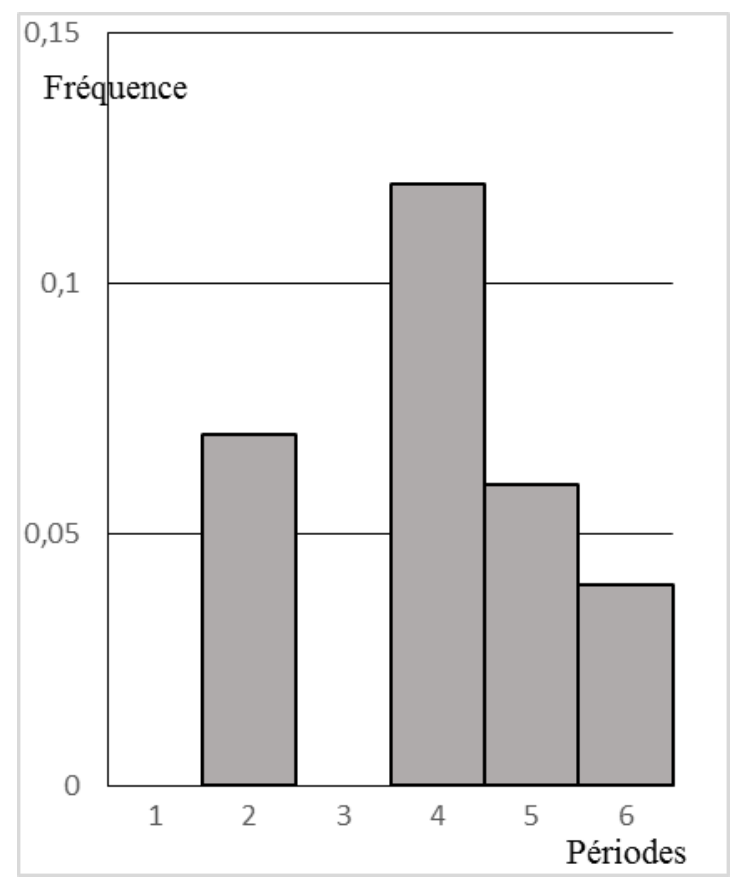

Figure 1. Spectre de PCUS

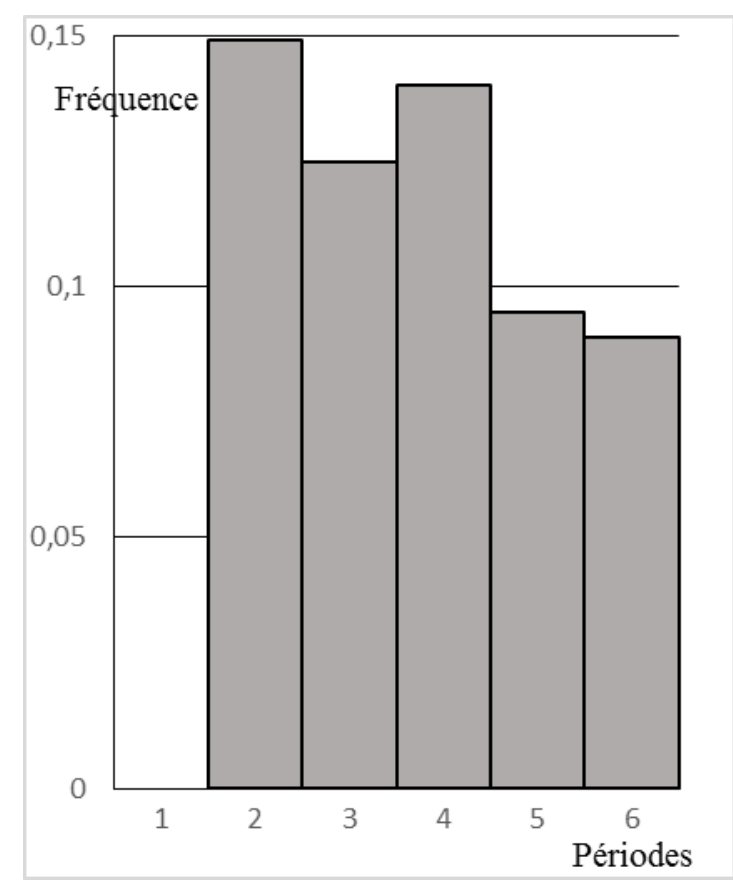

Figure 2. Spectre de Lénine

Quant au fameux « retour à Lénine » annoncé par le $\mathrm{XX}^{\mathrm{e}}$ Congrès, on en cherchera vainement trace dans les timbres. Enfin le tableau 3 indique clairement que ces thèmes sont tous deux des spécialités staliniennes. II est alors remarquable de constater la disparition totale du PCUS pendant la guerre mondiale (période 3), d'ailleurs baptisée par les soviétiques «grande guerre patriotique $»^{8}$.

Enfin la Révolution, les soviets, la guerre civile, sont largement présents encore sous Staline avant-guerre. De plus en plus largement associés au PC, à Lénine, à l'Armée rouge et même au visage de Staline, ce n'est bientôt plus qu'un fond de drapeaux rouges et une inscription en lettres d'or au bas du timbre. On est loin du premier timbre émis par la jeune République : un soleil levant et une épée qui brise les chaînes de la servitude (illustr. 4). Le thème se fait plus lointain et plus discret, par la quantité au moins (fig. 3).

Dans l'ensemble de la production postale, la prépondérance revient aux thèmes proprement soviétiques, nationaux.

\footnotetext{
${ }^{8}$ Il semble bien que l'effacement du Parti communiste pendant cette période ne soit pas simplement «postal ». Il pourrait s'agir d'un des traits les plus marquants de cette période : «J'ai parcouru récemment les collections de journaux de juillet à novembre 1941. Le nom de Staline y est à peine mentionné; pour la première fois, depuis de longues années, on n'y trouvait ni ses portraits, ni d'épithètes admiratives (...) Staline lui-même semblait avoir compris qu'il fallait s'effacer. » EHRENBOURG (I.), La Russie en guerre, Paris, Gallimard, 1968, pp. 16-17.
} 


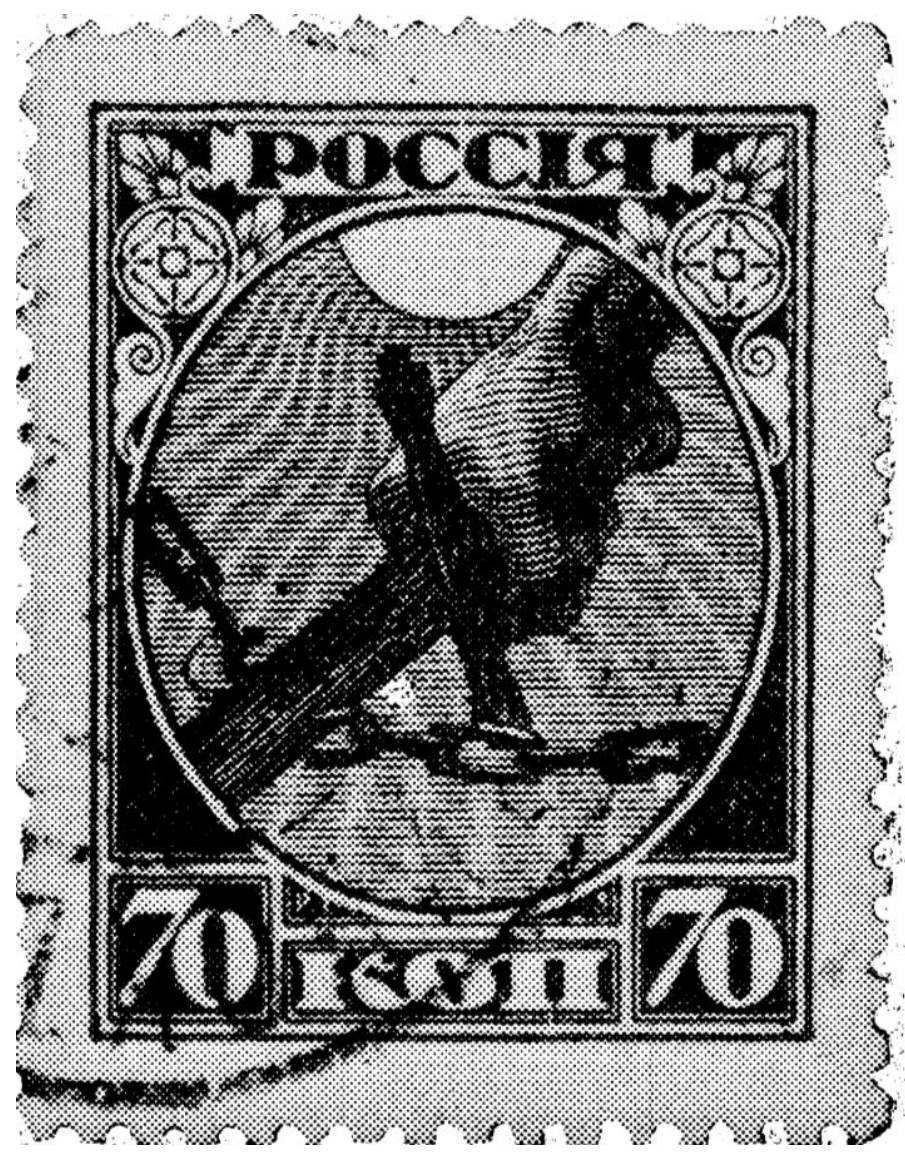

Illustration 4. Une épée brisant une chaîne. C'est le thème du premier timbre émis par l'Etat soviétique.

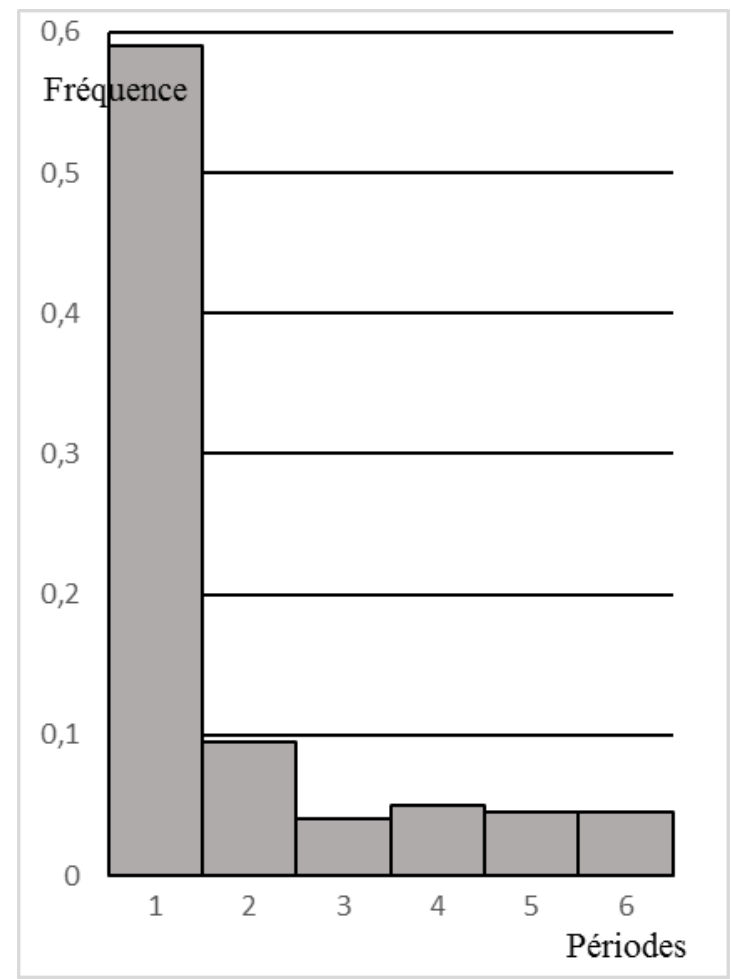

Figure 3. Spectre de Révolution d'octobre



Figure 4. Spectre de Moscou et l'URSS 


\section{Les thèmes soviétiques}

Dans ce deuxième groupe et par ordre d'importance numérique, on trouve les thèmes «Moscou-URSS » puis « économie nationale », « Armée rouge et décorations », « seconde guerre mondiale » enfin « histoire russe ».

Les paysages et monuments sont dans tous les pays un des motifs principaux choisis par les postes. L'URSS n'échappe pas à cette règle d'or puisque les timbres de ce type sont les plus nombreux (tableau 1). On retrouve la multiplicité des nationalités, des costumes, des mœurs et des pays constituants l'Union soviétique. Cette série d'images rappelle « La sixième partie du monde » de Vertov qui montre la diversité de l'URSS « aussi bien sous le cercle polaire, que dans les déserts du centre asiatique »(G. Sadoul). L'ensemble est toutefois fortement cimenté par l'existence de la capitale Moscou à qui est consacrée environ un tiers des timbres de ce thème. L'accent mis à la fois sur la diversité des nationalités et sur l'aspect centralisateur de la capitale peut expliquer pourquoi ce thème connaît ses plus fortes occurrences sous Staline avant et après-guerre (fig. 4). Au surplus, il n'aura pas échappé au philatéliste averti que l'ensemble des timbres est libellé en russe, à la différence, par exemple des billets de banque.

$L^{\prime}$ '« histoire russe », baptisée aussi « histoire de notre pays » quoiqu'il s'agisse d'un thème mineur, confirme cette impression. Il s'agit en effet de l'histoire proprement russe d'avant 1917 : guerres napoléoniennes, de Crimée, russo-japonaise, progressistes russes, etc. Y figurent même une douzaine de timbres commémorant le rattachement de l'Ukraine et d'autres territoires à l'Empire russe. Le spectre de ce thème montre sans ambiguïté que c'est sous la période stalinienne, y compris pendant la guerre, que cette légitimité « russe » a été le plus invoquée (fig. 5).

C'est sans étonnement qu'on voit figurer en troisième position dans le tableau 1 , le thème «économie nationale ». Plutôt que d'économie, il s'agit d'ailleurs surtout d'industrie et même, jusque récemment, d'industrie lourde (40\% du total du thème). Ces séries affectionnent les décors d'usines gigantesques, d'aciéries, de coulées, de machines énormes, gloires de l'Union soviétique pendant toute une époque (illustr. 5), Les ouvriers au travail ont des poses héroïques. Toute l'imagerie, volontiers lyrique, des années trente à cinquante défile ici comme elle s'affichait sur les murs et s'étalait dans les revues. 


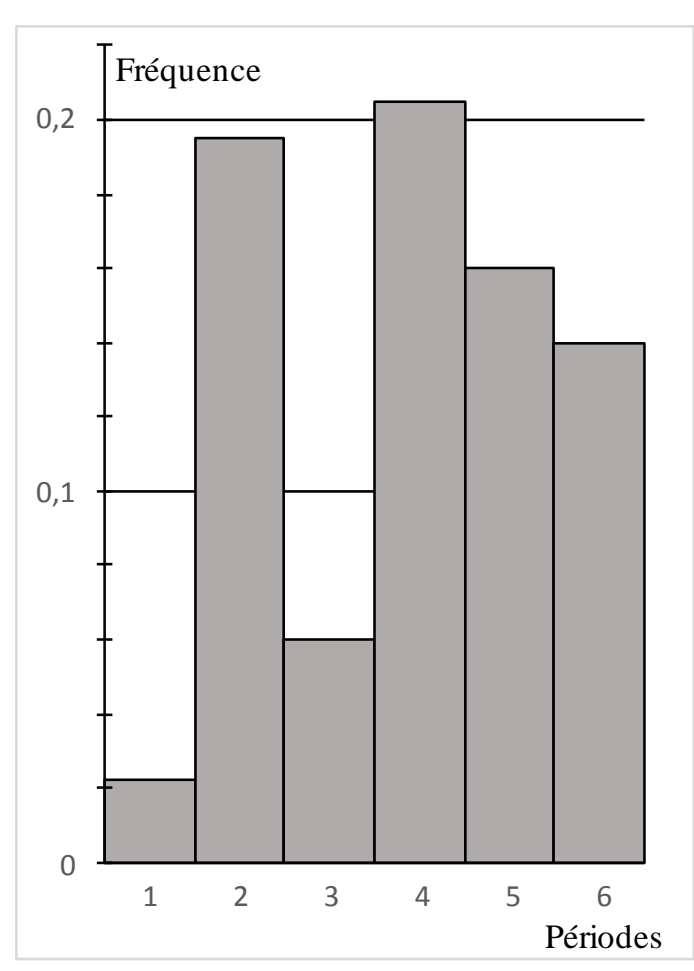

Figure 5. Spectre de Histoire russe

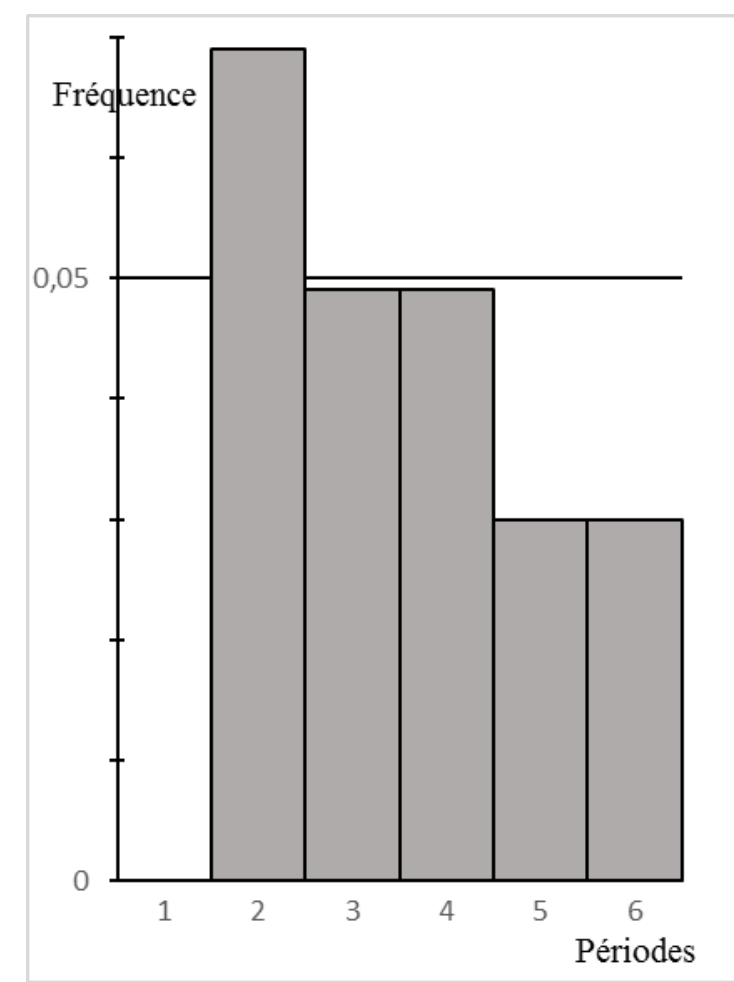

Figure 6. Spectre de Economie

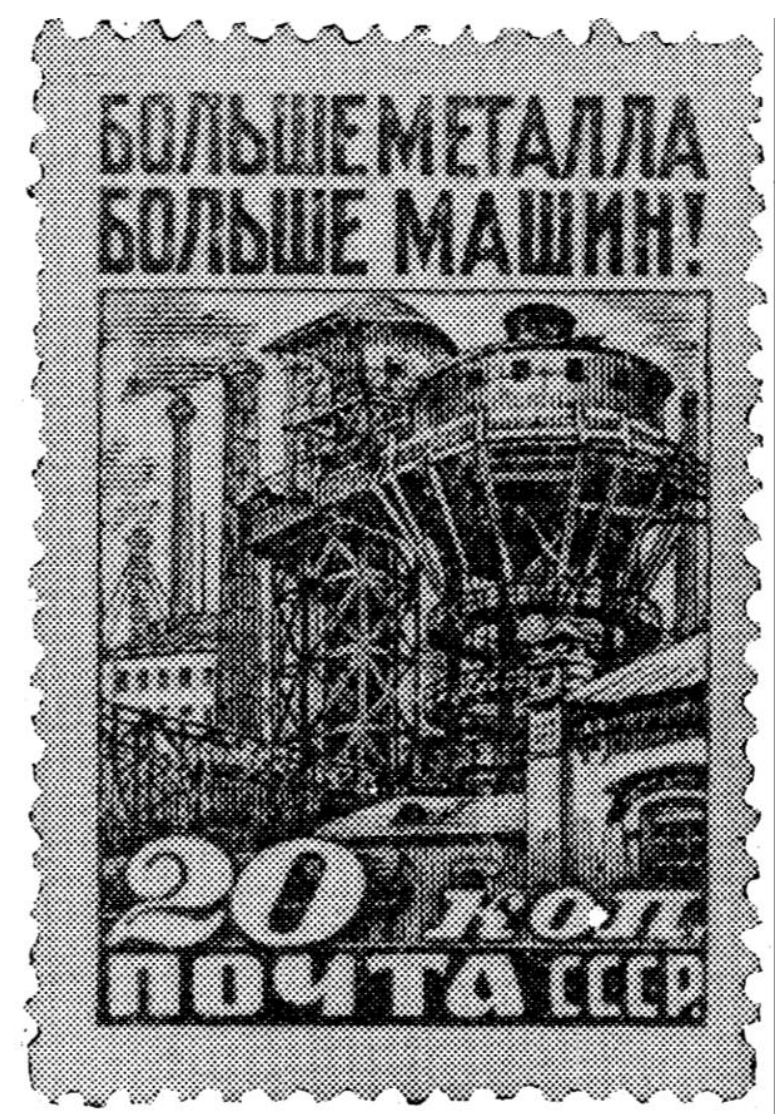

Illustration 5. «Davantage de métal, davantage de machines ! » proclame ce timbre émis dans les années 30. 
Après l'industrie, un quart des timbres sont consacrés aux transports. L'effort gigantesque accompli par les soviétiques dans ce domaine transparaît abondamment dans le corpus. Cette préoccupation ne se limite pas aux chemins de fer et s'étend aux voies maritimes, aériennes et garde aujourd'hui son actualité comme le prouve, par exemple, l'image de cet hélicoptère géant (illustr. 6), La paysannerie enfin, n'a jamais été oubliée, au moins dans ses aspects socialistes. Les kolkhoses, entre autres, ont fait l'objet de plusieurs séries. Elle représente un petit quart de ce thème, Là encore, il s'agît d'une spécialité de l'époque stalinienne comme l'indiquent à la fois l'indicateur binaire (présence forte en 1924-1941 et 1945-1956) et le profil du spectre qui n'est pas sans rappeler étrangement celui de Lénine (comparer les fig. 2 et 6).

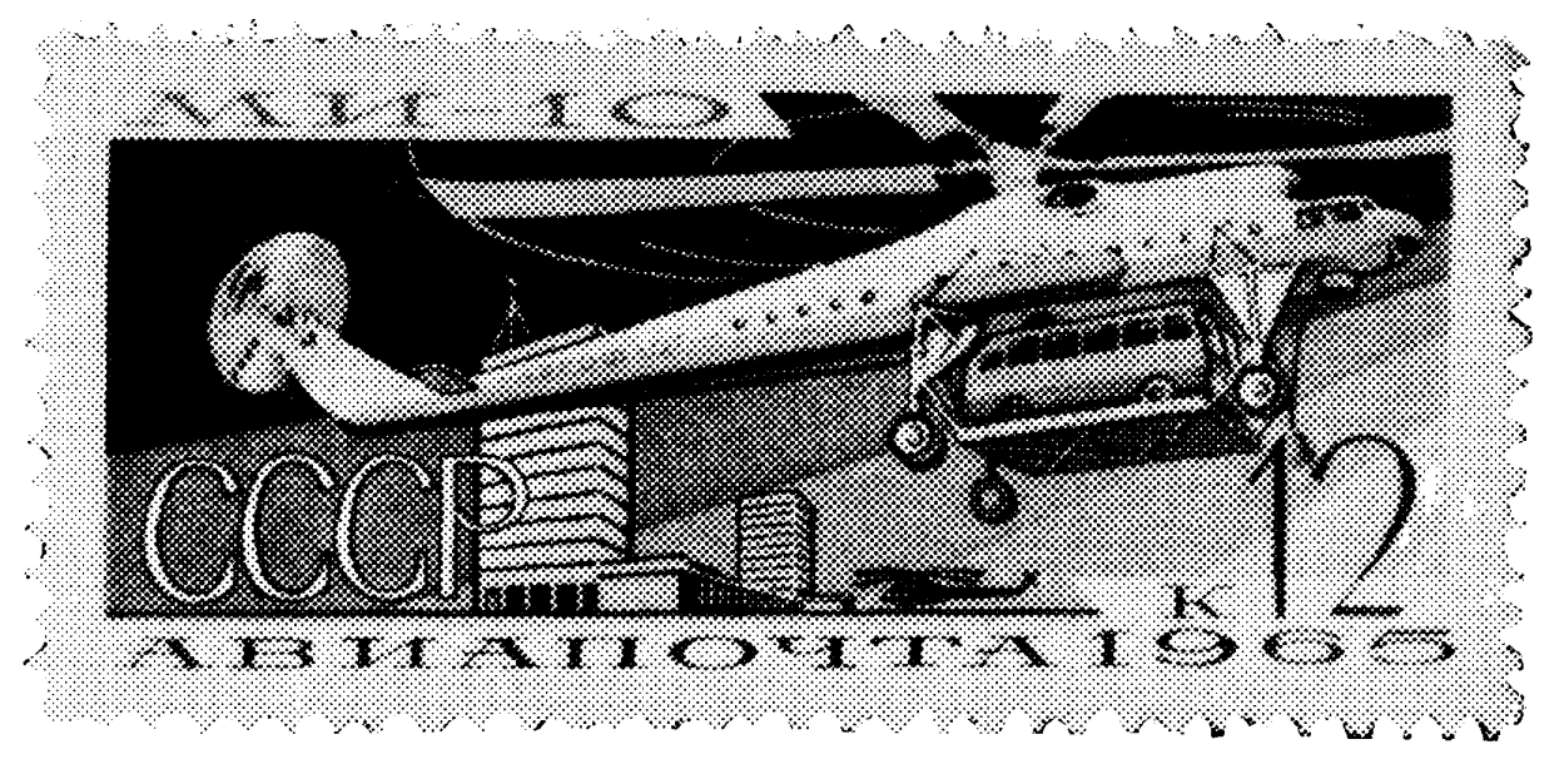

Illustration 6. Ce timbre représente l'hélicoptère géant Ml 10 (1965).

Il est probable qu'il faut y voir une sorte de légitimation à deux degrés. Lénine serait la source de légitimité de l'Etat et du PCUS et l'industrialisation entreprise à partir de 1928, le but suprême poursuivi par eux.

Deux autres thèmes typiquement patriotiques encore, «l'Armée rouge et les décorations », et la « grande guerre patriotique »(1941-1945). L'Armée rouge connaît un sort inverse à la Révolution d'Octobre. Au fur et à mesure que celle-ci s'estompe, l'Armée et les thèmes guerriers prennent de plus en plus de place et ceci dès avant 1941. Trois remarques s'imposent à propos de ces thèmes.

L'armée est un aspect typique de la société stalinienne. Il est notable que les « ordres et décorations » soient largement prépondérants. Le premier timbre de cette série date de 1933 et c'est à partir de 1941 que le catalogue se remplit de médailles et d'ordres nouveaux qui composent le plus clair de la production vers la fin de la guerre. Auparavant, il s'agissait plutôt de tableaux guerriers, d'armes, etc. 


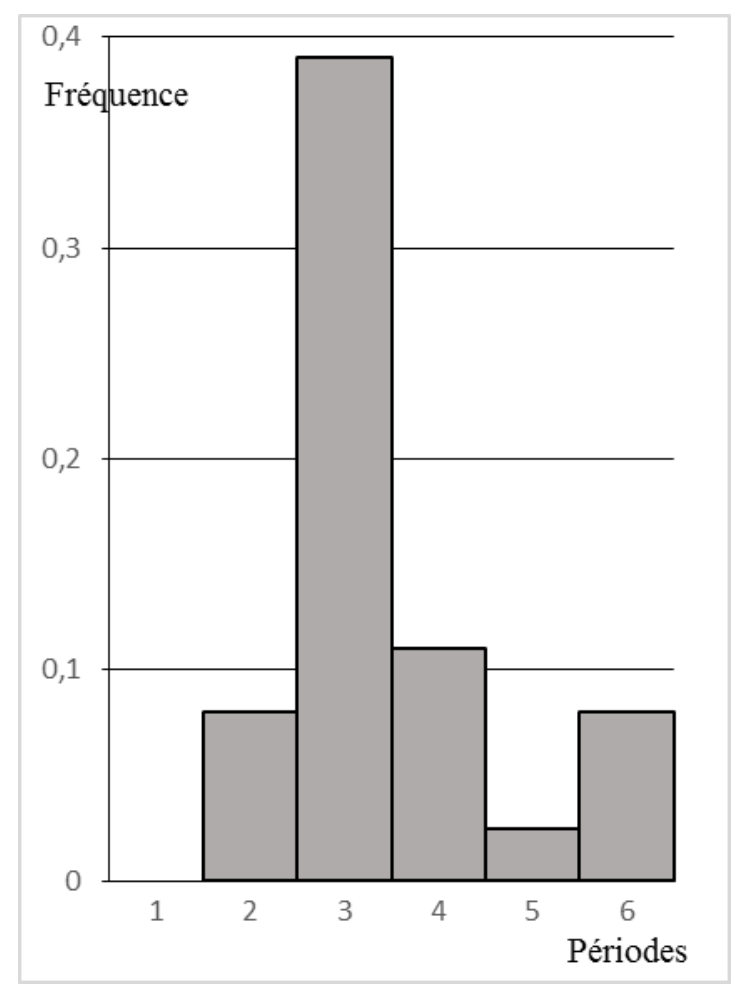

Figure 7. Spectre de Armée et décorations



Figure 8. Spectre de Seconde guerre mondiale

Ce thème connaît un creux tout à fait remarquable sous Khrouchtchev, époque où le poète Evtouchenko écrivait : «Les russes veulent-ils la guerre ? ». C'est le dégel et la détente qui se manifestent par ailleurs et, entre autres, par une forte croissance des « relations internationales ».

Tout aussi remarquable est la croissance rapide du thème "seconde guerre mondiale » après la chute de Khrouchtchev (fig. 8). Tout en gardant volontiers un style humaniste (voir par exemple la «ballade du soldat », illustr. 7), les thèmes patriotiques issus de la guerre semblent rappeler aux soviétiques qu'une guerre est toujours possible sans que le visage de l'ennemi potentiel soit bien explicite.

\section{Les thèmes généraux}

Dans ce dernier groupe, à première vue plus hétéroclite, sont regroupés des thèmes dont l'aspect politique ou national n'est pas évident a priori. Mais alors que dans les deux parties précédentes la réalité et l'histoire se traduisaient idéologiquement dans le timbre, on se trouve ici plutôt face à une traduction idéologique de l'idéologie. Ce phénomène trouvant naturellement son champ d'action privilégié dans la littérature et l'art, deuxième thème par son importance numérique.

Pour la littérature, plus des quatre cinquième des timbres portent sur des auteurs et des œuvres soviétiques et en grande majorité, russes. Le reste allant à des auteurs étrangers. Parmi eux citons les français : Victor Hugo, Béranger, J.-J. Rousseau, Diderot, R. Rolland, Eugène 
Pottier, Voltaire. L'art trouve également sa source principale dans le fonds commun des œuvres russes et des autres peuples soviétiques.

C'est le thème majeur de la Révolution d'Octobre qui traduit à sa manière l'immense mouvement intellectuel de cette époque. Il devient plus discret et son contenu plus classique sous Staline (avant et après-guerre). De 1941 à 1945 et dans la période khrouchtchévienne, il connaît une faveur à peine plus grande (fig. 9).

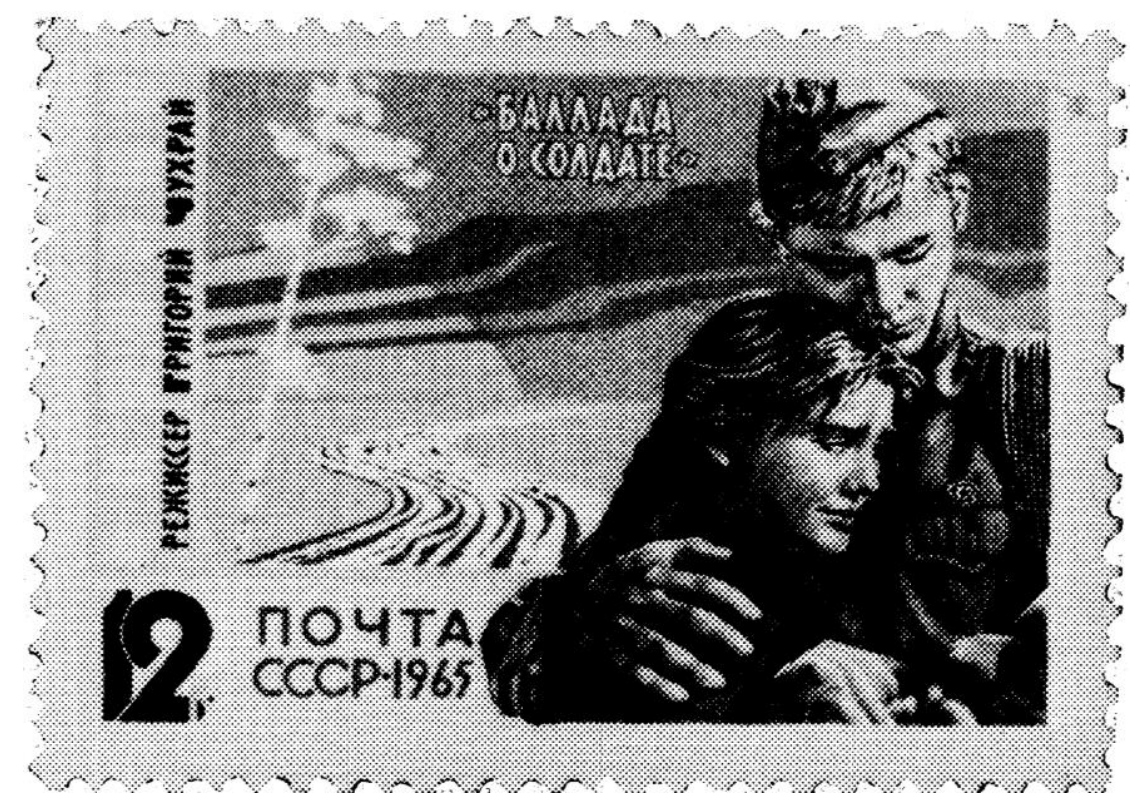

Illustration 7. Le timbre consacré au film de Grigori Tchoukraï, La Ballade du soldat (1965).

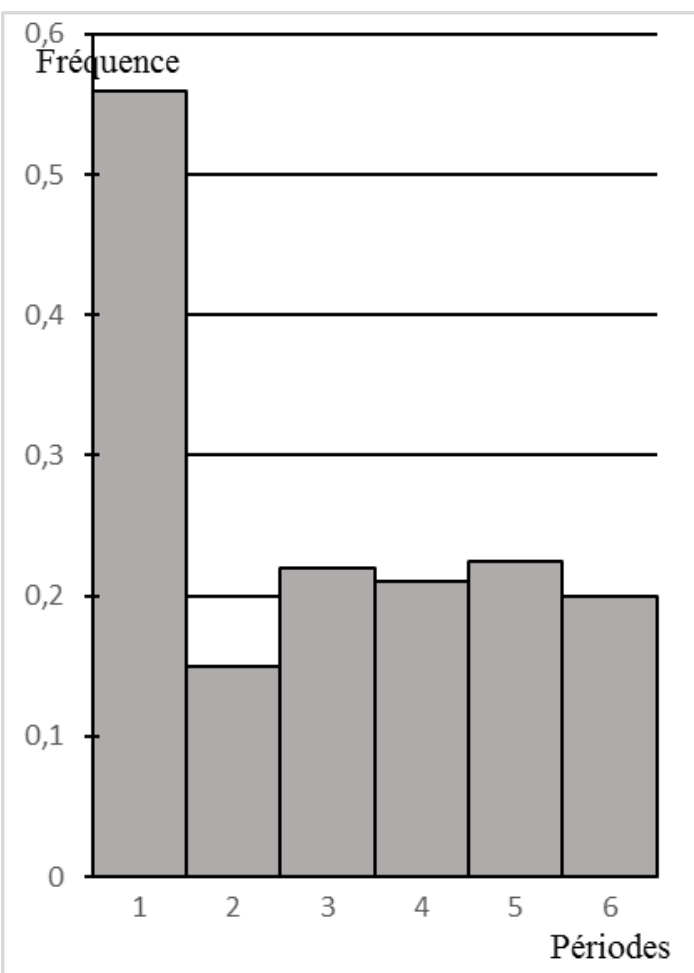

Figure 9. Spectre de Littérature et arts

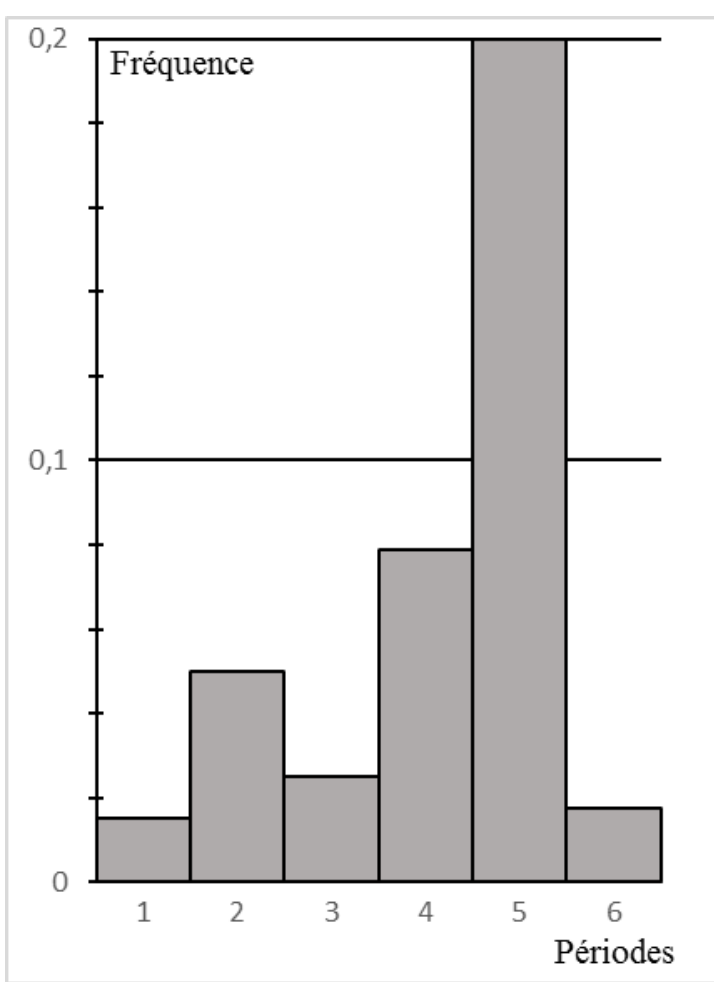

Figure 10. Spectre de Relations internationales 
Les relations internationales sont une catégorie assez hétérogène. Dans le catalogue philatélique, cette rubrique regroupe à la fois le mouvement communiste et ouvrier, les mouvements de libération nationale tout comme la coopération internationale dans son sens le plus large, y compris les relations commerciales. C'est ainsi que peuvent se côtoyer un timbre représentant $\mathrm{M}$. Thorez et un timbre sur le troisième congrès mondial de protozoologie. Image sans doute assez représentative de la façon dont les soviétiques ont souvent tendance à concevoir les relations avec l'étranger. Nous leur avons adjoint les timbres relatifs au bloc socialiste, quelques dizaines, parmi lesquels curieusement seule la Chine n'apparut jamais. Ce regroupement, d'incidence statistique négligeable, nous semble conforme à l'esprit de ce thème. Faible ou inexistant jusqu'au XXe Congrès, il connaît un bond fantastique sous Khrouchtchev, progression qui se dément à peine à partir de la chute de ce dernier (fig. 10).

Les sports que nous avions considérés a priori comme une catégorie autonome, suivent en fait une évolution extrêmement proche des relations internationales (fig. 11). Cela traduit probablement le rôle que l'Etat soviétique attribue au sport dans la compétition pacifique entre Etats (illustr. 8).

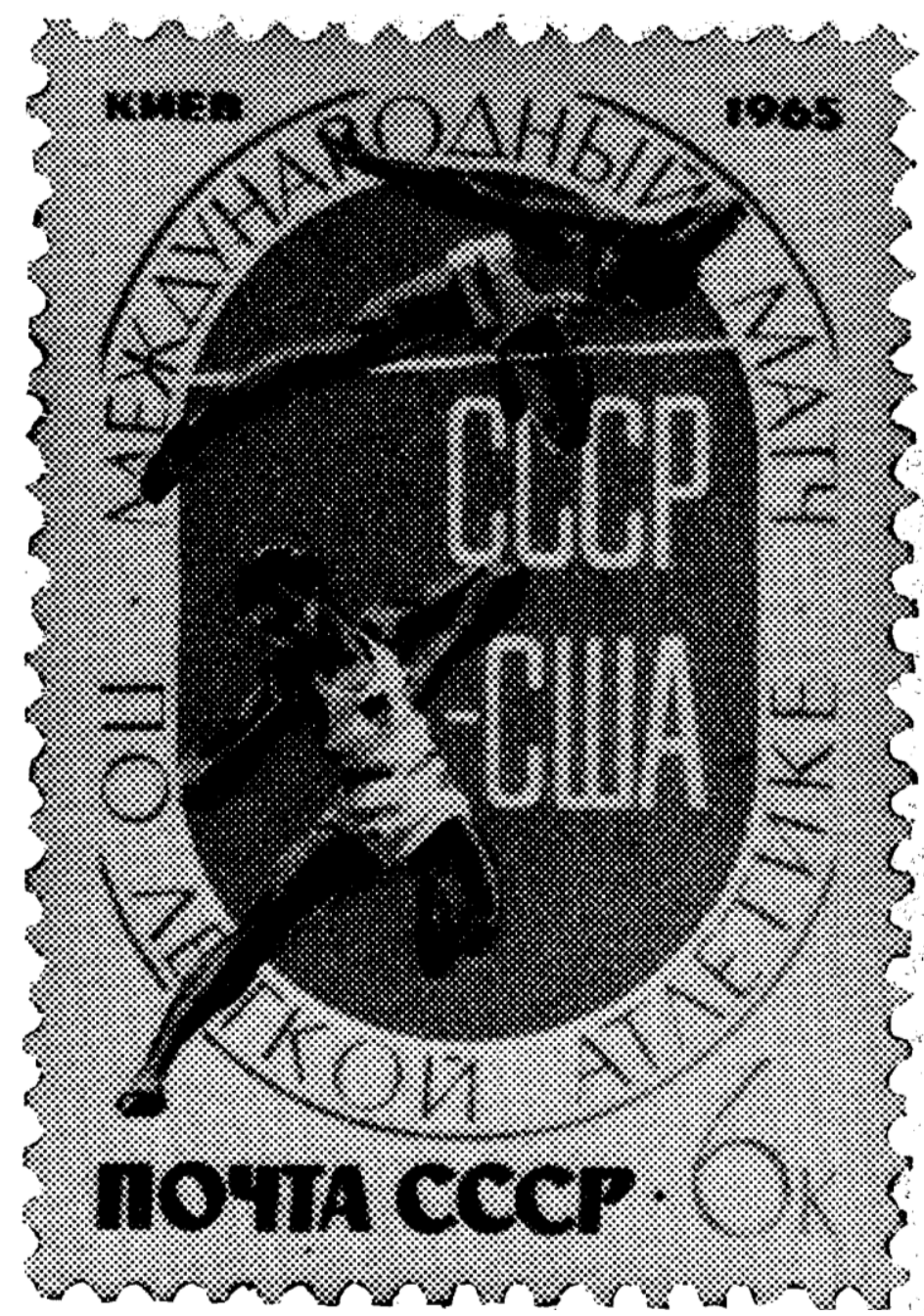

Illustration 8. Les sports : ce timbre commémore le match d'athlétisme USA-URSS qui se déroula à Kiev (1965). 


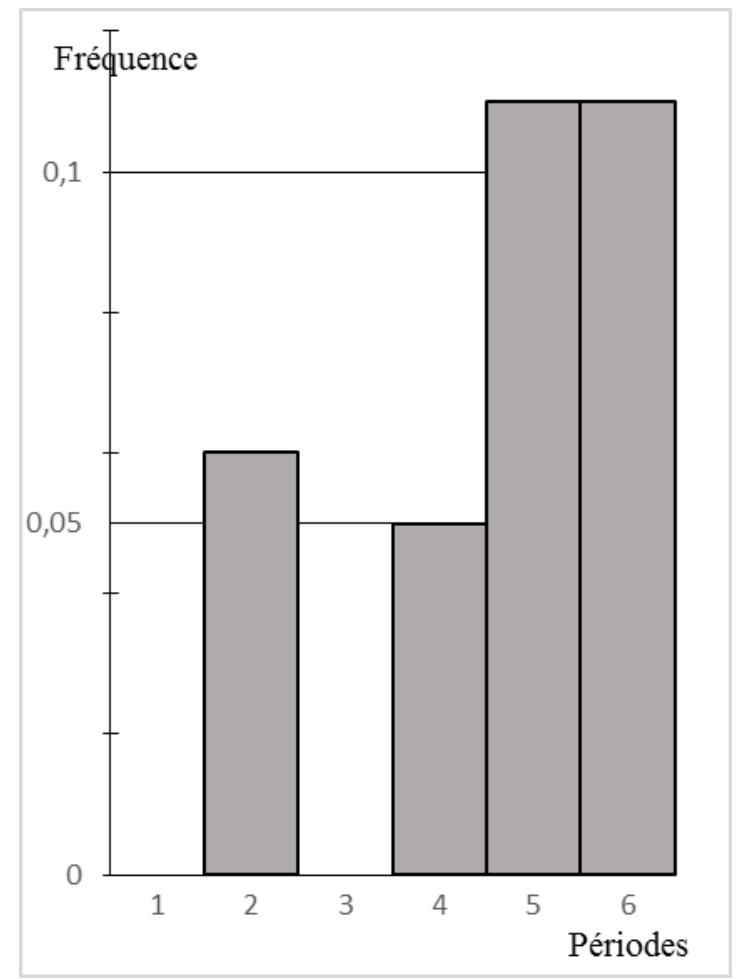

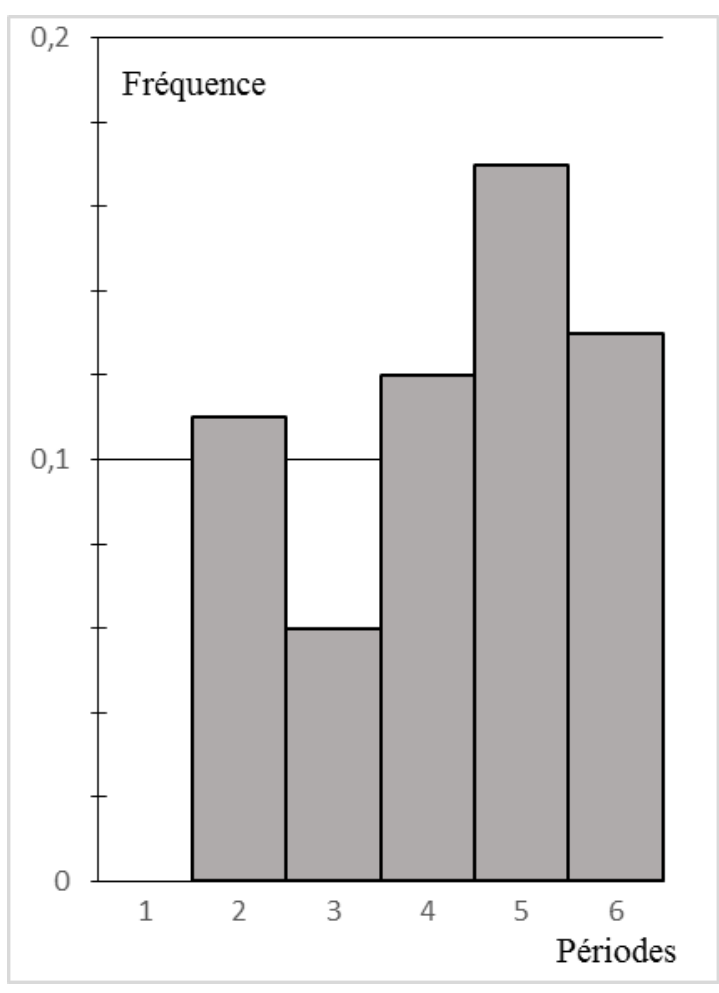

Figure 12. Spectre de Sciences et techniques

Figure 11. Spectre de Sports

L'évolution des «sciences et techniques » suit une ligne semblable (fig. 12), Elle est en effet le terrain privilégié de la compétition pacifique à partir de 1956. On peut penser qu'il s'agit là d'un reflet d'une mutation profonde de la société soviétique. Le gros de ces timbres est fourni par la conquête de l'espace. Ce serait au fond un changement d'objectifs de la société soviétique : on propose moins maintenant de produire plus d'acier, plus de machines mais plutôt de conquérir la suprématie dans les techniques de pointe (espace, atome, électronique, aéronautique, etc.) qui sont les nouveaux motifs dominants de ce thème (illustr. 9). Cette évolution «moderniste » se trouve confirmée par l'apparition en force, à partir de $1956 \mathrm{du}$ thème « Nature et environnement» (fig. 13 et illustr. 10) ${ }^{9}$.

\footnotetext{
${ }^{9}$ On aura certainement remarqué que 1956 coïncide avec une baisse sensible des thèmes « Lénine » et «PCUS ». Ce recul qui s'effectue en raison inverse des « sciences et techniques » est contemporain de l'apparition de la théorie de la « révolution scientifique et technique », et pourrait bien marquer une évolution vers un type de légitimité rationaliste et non plus révolutionnaire ou nationale comme auparavant.
} 


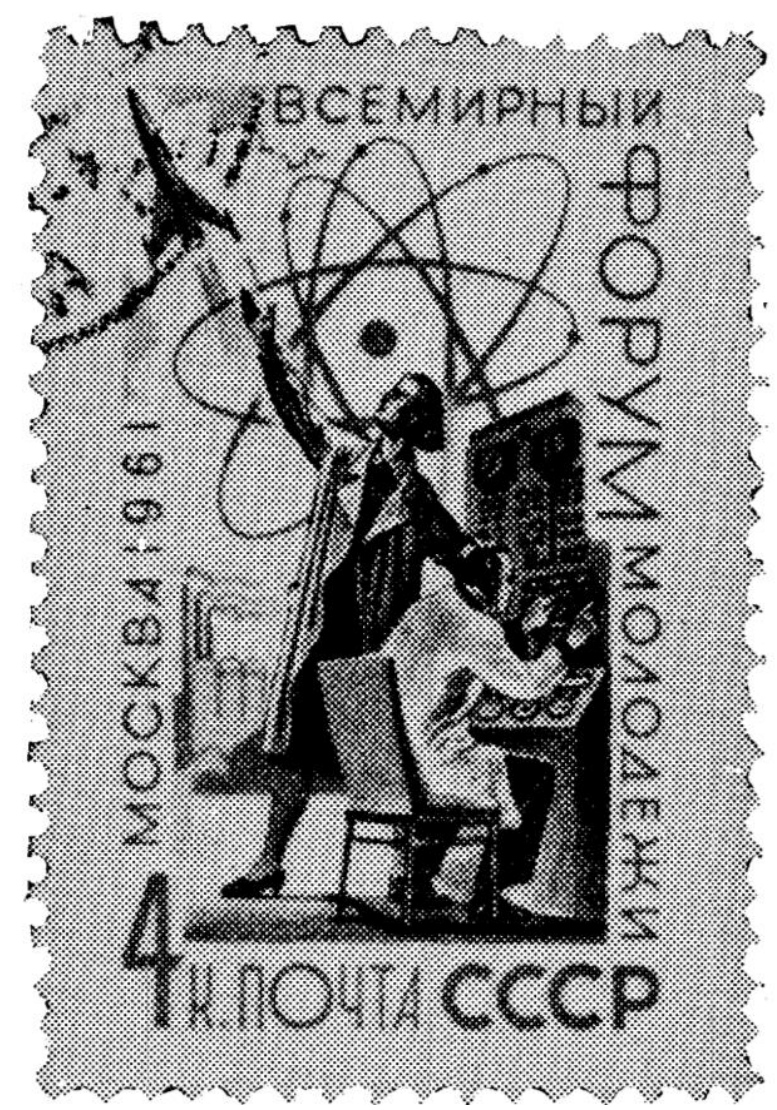

Illustration 9. L'imagerie de la science et des techniques sur un timbre consacré au Forum mondial de la jeunesse (1961).

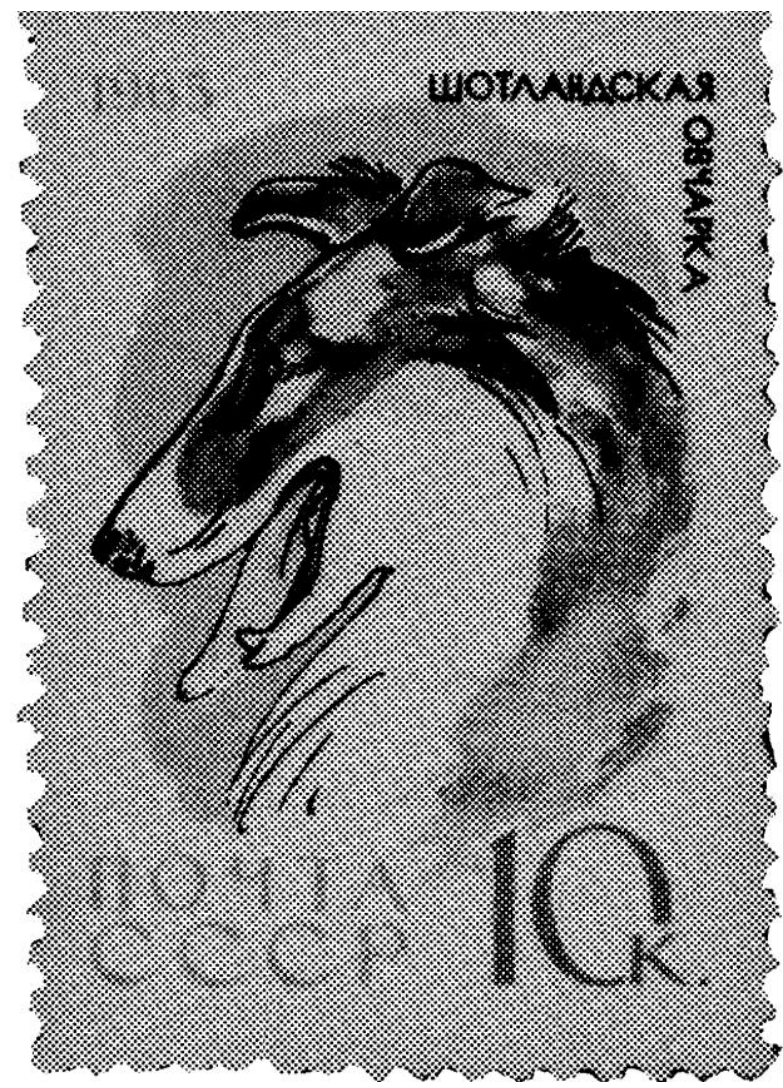

Illustration 10. Un chien de race illustre l'environnement (1965). 


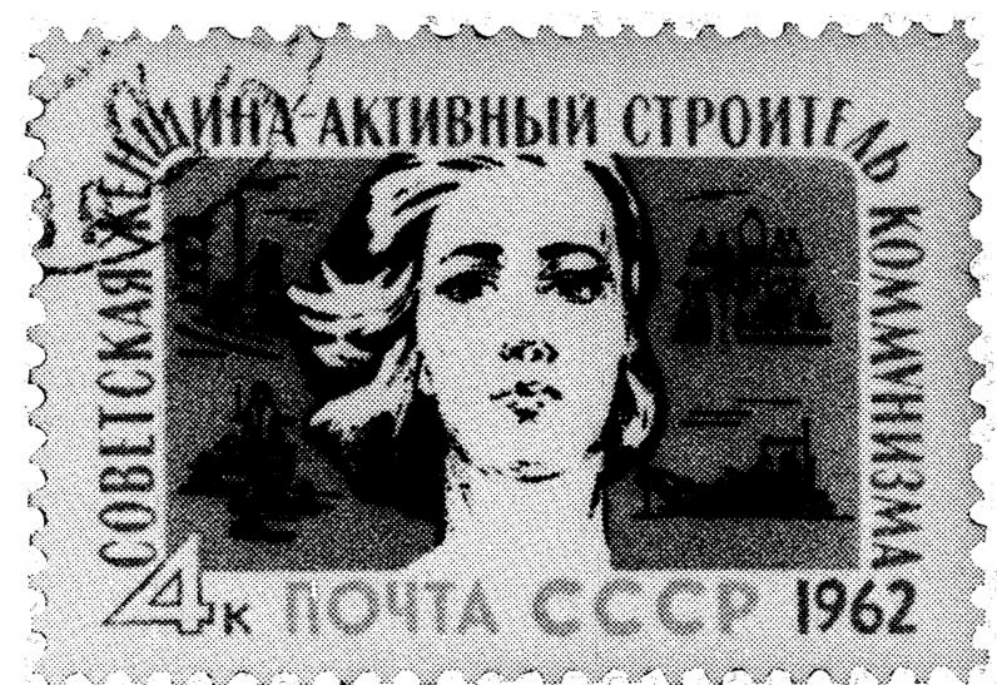

11. «La femme soviétique, constructrice du communisme » (1962).

Il est de coutume dans le monde soviétique de faire référence à certaines couches sociales spécifiques comme les femmes ou la jeunesse. Ces couches possèdent leurs organisations, journaux, manifestations ... elles ont aussi leurs timbres. Il est frappant de noter que ces deux couches ont été particulièrement célébrées pendant la période khrouchtchévienne (illustr. 11), la jeunesse ayant eu quant elle l'amer privilège d'être exaltée pendant la guerre (fig. 14 et 15).

$\mathrm{Au}$ total, donc, on observe une évolution historique assez nette quoique diverse et multiple. Pour tenter d'en saisir le sens général dans sa globalité nous avons construit un dernier tableau (tableau 4) qui, à partir des indicateurs binaires, mesure les taux de variation entre périodes historiques.

Tableau 4. Taux de variation entre période calculés à l'aide des indicateurs binaires

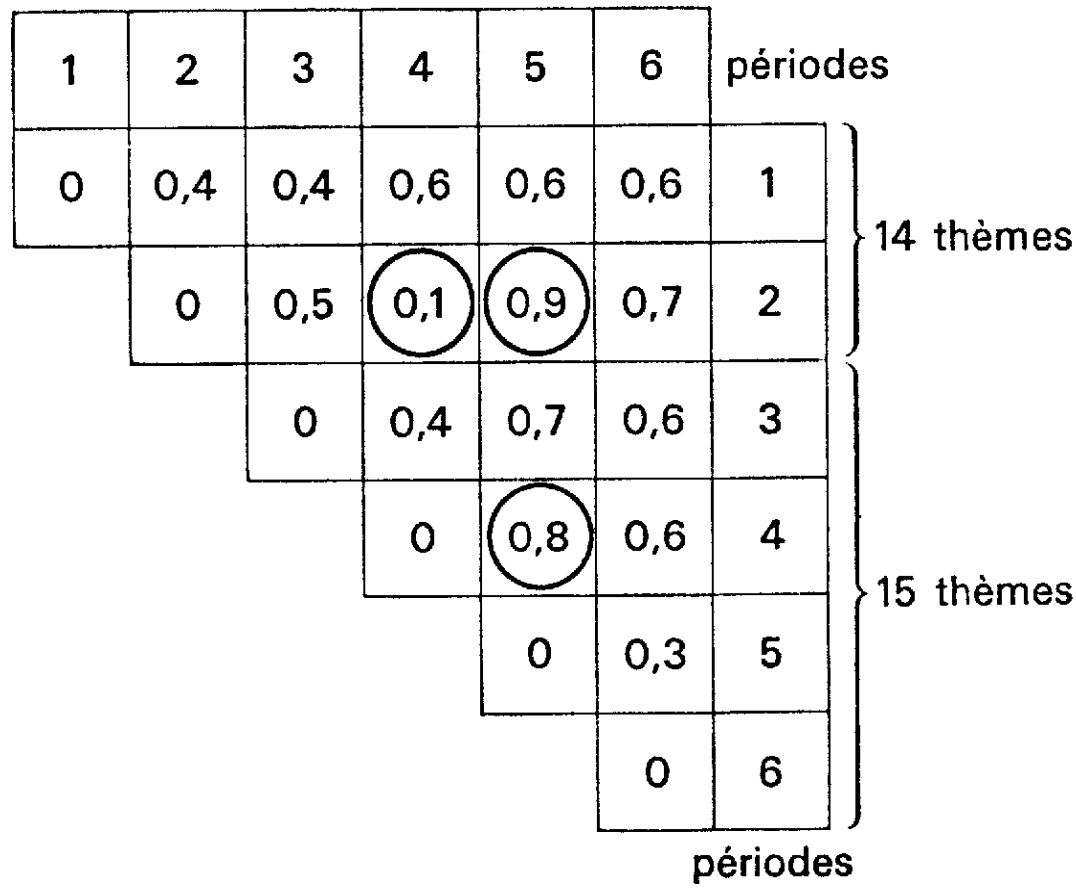


Si l'on se réfère à ce dernier tableau on peut constater que, au point du point de vue structural, les périodes les plus semblables sont d'une part, la deuxième et la quatrième (1924-1941 et 1944-1956), et d'autre part la cinquième et la sixième (1956-1964 et 1964-1969), ces deux groupes étant eux-mêmes fort dissemblables. La disparité est maximum entre la période de Staline avant et après-guerre et la période khrouchtchévienne (à 90 et $80 \%$ ). Enfin deux périodes se situent à part, celle qui suit Octobre jusqu'en 1924 et celle de la seconde guerre mondiale.

Cela nous confirme qu'en dehors de ces deux dernières périodes, qui sont tout à fait exceptionnelles, la coupure majeure de l'histoire soviétique pourrait se situer autour de l'époque allant de la mort de Staline au $\mathrm{XX}^{\mathrm{e}}$ Congrès. Certes, ceci ne constitue pas précisément une découverte, toutefois la netteté de la coupure constatée dans le corpus est un élément de validation de notre méthode.

Cet essai sans doute trop rapide et superficiel montre que la philatélie peut fournir un champ d'investigation assez riche pour le chercheur politique. L'arsenal mathématique que nous avons utilisé est certes relativement rustique mais suffisant pour une appréhension globale qui ne prétend pas épuiser tel ou tel problème spécifique. 